\title{
MAC Layer Performance Analysis of Dense Small Cell Networks with Full Duplex
}

\author{
(Invited Paper) \\ Tian Ding $\sharp$, Ming Ding ${ }^{\ddagger}$, Guoqiang Mao ${ }^{\sharp \ddagger}$ \\ ${ }^{\sharp}$ School of Electrical and Data Engineering, University of Technology Sydney, Australia \\ ${ }^{\ddagger}$ Data61, Australia
}

\begin{abstract}
The study of full duplex (FD) is important because it has been identified as one of the candidate technologies for the 5th-generation (5G) networks. Small cell networks (SCNs) are envisioned to embrace the FD transmission technology in order to increase the spectral efficiency of wireless systems. In this paper, for the first time, we consider FD communications in a realistic small cell networks (SCN) scenario, where base stations (BSs) can select FD or half-duplex (HD) mode according to the real-time downlink (DL)/ uplink (UL) traffic. We present analytical results on the probabilities of BS mode selection, which match the simulation results well.
\end{abstract}

\section{INTRODUCTION}

The orthogonal deployment of dense small cell networks (SCNs) with the existing macrocell networks implies that small cells and macrocells operate on different frequency spectrum. Due to its capacity gain and easy implementation, such deployment has been selected as the workhorse for capacity enhancement in the 4th-generation $(4 \mathrm{G})$ and 5 thgeneration $(5 \mathrm{G})$ networks developed by the 3rd Generation Partnership Project (3GPP) [1]. It is also envisaged that dense SCNs will embrace full duplex (FD), which is the successor of the existing half-duplex (HD), e.g., dynamic time division duplex (TDD). Moreover, the FD communications has been identified as one of the candidate $5 \mathrm{G}$ technologies.

With FD, both downlink (DL) and uplink(UL) can operate simultaneously in each base station (BS). Compared with HD, the implementation of FD introduces additional interference to the networks, i.e., the intra-cell interference and the intercell interference. From the aspect of intra-cell interference, the UL of FD BSs and/or user equipments (UEs) suffer from self-interference (SI) due to the DL signal leakage resulting from the imperfect isolation between transmit and receive antennas. Moreover, the HD UEs in a FD cell are affected by the intra-cell inter-link interference. From the aspect of intercell interference, the inter-cell inter-link interference increases because the number of interfering links increase. Besides this challenge in the physical (PHY) layer of FD, it is important to investigate the media access control (MAC) layer performance of FD. To the best our knowledge, the MAC layer analysis of FD has not been investigated from a theoretical viewpoint in the literature.

In [2], the authors assumed a heterogeneous $\mathrm{SCN}$ scenario with FD, where the small BSs (SBSs) worked in FD mode and the UEs worked in HD mode. In [3], the authors considered a hybrid duplex SCN scenario, where the BSs could select
FD/HD mode and the UEs worked in HD mode. In [4], the authors studied the performance of FD multiple-input multiple-output (MIMO) SCN, where the BSs worked in FD mode and the UEs worked in HD mode. Most previous works only assumed HD UE because it is difficult for UE to perform self-interference cancellation (SIC). However, recent technology has shown that the UE has potential in reducing the SI to the level of noise power [5], which makes it possible for manufacturing the FD UE. Moreover, the process of replacing HD UE with FD UE may take a long period of time. As a result, it is anticipated that the FD UEs will coexist with the HD UEs in a FD SCN. Therefore it is interesting to investigate the performance of SCN with a hybrid of FD BSs, FD UEs and HD UEs.

In this paper, for the first time, we conduct a theoretical study on the MAC layer performance of FD transmission technology for a realistic SCN scenario, where BSs can select FD/HD mode according to the real-time DL/UL traffic. More specifically, we focus on a fundamental question: how will the network densification and the process of replacing HD UE with FD UE affect the MAC layer performance of SCN with FD? To answer this question, we present a single contribution in this paper as follows:

- We derive closed-form expressions for the probabilities of BS mode selection, considering a realistic SCN scenario with a hybrid of FD BSs and FD/HD UEs.

\section{SySTEM MOdeL}

\section{A. Network Scenario}

In this subsection, we present the network scenario considered in the paper. Note that in this paper we only focus on the MAC layer performance analysis, and the considered network scenario is used to derive the distribution of the UE number in an active BS, which will be shown in Subsection III-A. The PHY layer performance analysis will be left as our future work.

We consider a cellular network with BSs deployed on a plane according to a homogeneous Poisson point process (HPPP) $\Phi$ with a density of $\lambda$ BSs $/ \mathrm{km}^{2}$. Active UEs are also Poisson distributed in the considered network with a density of $\rho \mathrm{UEs} / \mathrm{km}^{2}$. Here, we only consider active UEs in the network because non-active UEs do not trigger data transmission, and thus they are ignored in our analysis. 
In practice, a BS will mute its transmission if there is no UE connected to it, which reduces inter-cell interference and energy consumption [6]. Note that such BS idle mode operation is not trivial, which even changes the capacity scaling law [7]. Since UEs are randomly and uniformly distributed in the network, we assume that the active BSs also follow an HPPP distribution $\tilde{\Phi}$ [8], the density of which is denoted by $\tilde{\lambda} \mathrm{BSs} / \mathrm{km}^{2}$. Note that $0 \leq \tilde{\lambda} \leq \lambda$, and a larger $\rho$ leads to a larger $\tilde{\lambda}$. From $[8,9], \tilde{\lambda}$ is given by

$$
\tilde{\lambda}=\lambda\left[1-\frac{1}{\left(1+\frac{\rho}{q \lambda}\right)^{q}}\right],
$$

where $q$ takes an empirical value around 3.5 4 [8,9].

\section{B. BS Mode Selection}

In this subsection, we introduce the scheme of BS mode selection with FD. We consider a realistic SCN scenario with a hybrid of FD BSs and FD/HD UEs, where all the SBSs are capable of FD communications, and the UEs are composed of a coexistence of both FD UEs and HD UEs. The active UEs may request FD, HD-DL or HD-UL type of data, and the serving BSs select mode according to the real-time DL/UL traffic. For the considered network with a hybrid of FD BSs and FD/HD UEs, we have the following three remarks.

Remark 1: For an active FD UE (FU), it may not always request FD data. When a FD UE requests DL or UL data, it can be deemed as a HD UE.

Remark 2: For an active HD UE (HU), it can also request FD data, where it demands both DL and UL data simultaneously. Since the HD UE is not capable of FD communications, it may be scheduled with another FD-HU, DL or UL data for FD communications. If there is only one active UE in the cell, and the active UE is a HD UE requesting FD data, the HU is assumed to have priority of DL communications.

Remark 3: For an active BS, it can work in FD mode only if there exists at least one FD UE requesting FD data, or there exists at least one DL-UL data pair. The DL-UL data pair is formed by one DL data request and one UL data request, or by one FD data request from a HD UE and one DL/UL data request. Otherwise the active $\mathrm{BS}$ will work in HD mode.

We define the four BS working modes as follows.

1) FD BS with Single FD UE (FS): For an active BS, it will work in FS mode if there exists at least one FD UE requesting FD data. Note that we assume the FU requesting FD data has priority over the DL-UL data pair.

2) FD BS with DL-UL UE Pair (FP): For an active BS, it will work in FP mode if there exists at least one DL-UL data pair, and there is no FU requesting FD data.

3) HD BS with a DL UE (HD): For an active BS, it will work in HD mode if there only exists DL data; or there is only one active UE in the cell, and the active UE is a HD UE requesting FD data, as stated in Remark 2.

4) $\mathrm{HD} B S$ with an UL UE (HU): For an active BS, it will work in HU mode if there only exists UL data.

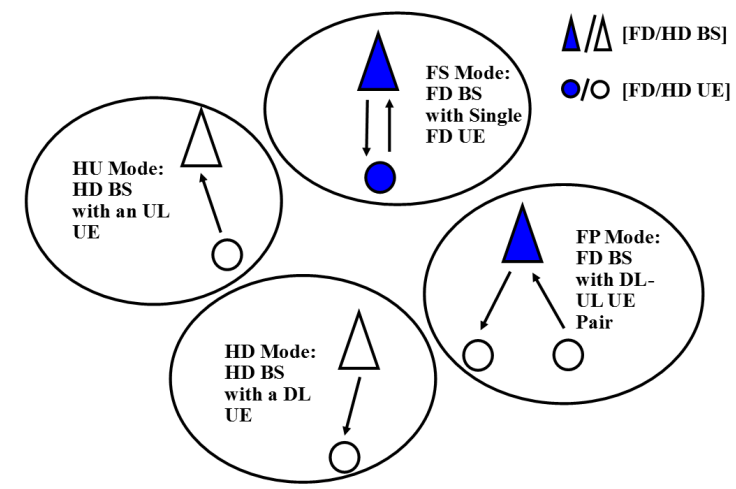

Fig. 1. A sketch of the four BS working modes.

Table I

NOTATION OF VARIABLES

\begin{tabular}{|c|c|}
\hline Notation & Items \\
\hline \hline$p^{\mathrm{FU}}, p^{\mathrm{HU}}$ & FD UE and HD UE ratios \\
\hline$p^{\mathrm{FF}}, p^{\mathrm{DF}}, p^{\mathrm{UF}}$ & $\begin{array}{c}\text { FD data, HD-DL data and HD-UL data } \\
\text { request probabilities of FD UE }\end{array}$ \\
\hline$p^{\mathrm{FH}}, p^{\mathrm{DH}}, p^{\mathrm{UH}}$ & $\begin{array}{c}\text { FD data, HD-DL data and HD-UL data } \\
\text { request probabilities of HD UE }\end{array}$ \\
\hline$q^{\mathrm{FS}}$ & $\begin{array}{c}\text { The probability of an active BS selecting } \\
\text { the FD mode with single UE }\end{array}$ \\
\hline$q^{\mathrm{FP}}$ & $\begin{array}{c}\text { The probability of an active BS selecting } \\
\text { the FD mode with a UE pair }\end{array}$ \\
\hline$q^{\mathrm{HD}}$ & $\begin{array}{c}\text { The probability of an active BS selecting } \\
\text { the HD DL mode }\end{array}$ \\
\hline$q^{\mathrm{HU}}$ & $\begin{array}{c}\text { The probability of an active BS selecting } \\
\text { the HD UL mode }\end{array}$ \\
\hline
\end{tabular}

A sketch of the four BS working modes is shown in Fig. 1. Note that in this paper we focus on the MAC layer performance analysis. Therefore the modelling and analysis of the PHY layer interference will be left as our future work.

\section{Performance Metrics}

The propotions of FD UE and HD UE are denoted as $p^{\mathrm{FU}}$ and $p^{\mathrm{HU}}$ respectively, where $p^{\mathrm{FU}}+p^{\mathrm{HU}}=1$. For each active FD UE, the probabilities of requesting FD data, DL data and $\mathrm{UL}$ data are respectively denoted by $p^{\mathrm{FF}}, p^{\mathrm{DF}}$ and $p^{\mathrm{UF}}$, where $p^{\mathrm{FF}}+p^{\mathrm{DF}}+p^{\mathrm{UF}}=1$. For each active HD UE, the probabilities of requesting DL data and UL data are respectively denoted by $p^{\mathrm{FH}}, p^{\mathrm{DH}}$ and $p^{\mathrm{UH}}$, where $p^{\mathrm{FH}}+p^{\mathrm{DH}}+p^{\mathrm{UH}}=1$.

We denote the working mode of an active BS by a string variable $Q$, where $Q$ takes the value of 'FS', 'FP', 'HD' and 'HU'. For clarity, 'FS', 'FP', 'HD' and 'HU' denote the FD mode with single UE, FD mode with a UE pair, HD DL mode and HD UL mode respectively. The probability of an active BS selecting the mode of $Q$ are denoted by $q^{Q}$. In the following sections, we will investigate the performance of $q^{Q}$. The notation of variables is summarized in Table I. 


\section{MAIN RESUlts OF MAC LAYER ANALysis}

\section{A. The Distribution of the UE Number in an Active BS}

Considering both active BSs and inactive BSs, the coverage area size $X$ can be characterized by a Gamma distribution [8]. Thus, the PDF of $X$ can be expressed by

$$
f_{X}(x)=(q \lambda)^{q} x^{q-1} \frac{\exp (-q \lambda x)}{\Gamma(q)},
$$

where $q$ is a distribution parameter and $\Gamma(\cdot)$ is the Gamma function [10].

Then, we denote the UE number per BS by a random variable (RV) $K$, and the probability mass function (PMF) of $K$ can be derived as

$$
\begin{aligned}
f_{K}(k) & =\operatorname{Pr}[K=k] \\
& \stackrel{(a)}{=} \int_{0}^{+\infty} \frac{(\rho x)^{k}}{k !} \exp (-\rho x) f_{X}(x) d x \\
& \stackrel{(b)}{=} \frac{\Gamma(k+q)}{\Gamma(k+1) \Gamma(q)}\left(\frac{\rho}{\rho+q \lambda}\right)^{k}\left(\frac{q \lambda}{\rho+q \lambda}\right)^{q},
\end{aligned}
$$

where (a) is due to the HPPP distribution of UEs and (b) is obtained from (2). It can be seen from (3) that $K$ follows a Negative Binomial distribution [10], i.e., $K \sim \mathrm{NB}\left(q, \frac{\rho}{\rho+q \lambda}\right)$.

As discussed in Subsection II-A, we assume that a BS with $K=0$ is not active, which will be ignored in our analysis due to its muted transmission. Hence, we focus on the active BSs and further study the distribution of the UE number in an active BS. For clarity, the UE number in an active BS is denoted by a positive RV $\tilde{K}$. Considering (3) and the fact that the only difference between $K$ and $\tilde{K}$ lies in $\tilde{K} \neq 0$, we can conclude that $\tilde{K}$ should follow a truncated Negative Binomial distribution, i.e., $\tilde{K} \sim \operatorname{truncNB}\left(q, \frac{\rho}{\rho+q \lambda}\right)$. More specifically, the PMF of $\tilde{K}$ is denoted by $f_{\tilde{K}}(\tilde{k}), \tilde{k} \in\{1,2, \ldots,+\infty\}$, and it is given by

$$
f_{\tilde{K}}(\tilde{k})=\operatorname{Pr}[\tilde{K}=\tilde{k}]=\frac{f_{K}(\tilde{k})}{1-f_{K}(0)},
$$

where the denominator $\left(1-f_{K}(0)\right)$ represents the probability of a BS being active. Note that based on the definition of $\tilde{\lambda}$ in Subsection II-A, we have $\left(1-f_{K}(0)\right)=\frac{\tilde{\lambda}}{\lambda}$.

\section{B. The Distribution of the Data Request Number in an Active BS: A Multinomial Distribution}

After obtaining $f_{\tilde{K}}(\tilde{k})$, we need to further study the distribution of the data request number in an active BS, so that the working mode of the BS can be determined in a FD network.

For clarity, the FD-FU, FD-HU, HD-DL and HD-UL data request numbers in an active $\mathrm{BS}$ are denoted by $\mathrm{RVs} M^{\mathrm{FFU}}$, $M^{\mathrm{FHU}}, M^{\mathrm{D}}$ and $M^{\mathrm{U}}$, respectively. Since we assume that each UE generates one request of FD data, DL data or UL data, it is easy to show that

$$
M^{\mathrm{FF}}+M^{\mathrm{FH}}+M^{\mathrm{D}}+M^{\mathrm{U}}=\tilde{K} .
$$

For each FD/HD UE in an active BS, the probability of it requesting $\mathrm{FD}$ data, DL data and $\mathrm{UL}$ data is $p^{\mathrm{FF}} / p^{\mathrm{FH}}$, $p^{\mathrm{DF}} / p^{\mathrm{DH}}$ and $p^{\mathrm{UF}} / p^{\mathrm{UH}}$, respectively. Therefore, for a given $p^{\mathrm{FU}}$, for each $\mathrm{UE}$ in an active $\mathrm{BS}$, the probability of it requesting FD-FU data, FD-HU data, DL data and UL data is $p^{\mathrm{FFU}}, p^{\mathrm{FHU}}, p^{\mathrm{D}}$ and $p^{\mathrm{U}}$, respectively, where $p^{\mathrm{FFU}}=p^{\mathrm{FU}} p^{\mathrm{FF}}$, $p^{\mathrm{FHU}}=p^{\mathrm{HU}} p^{\mathrm{FH}}, p^{\mathrm{D}}=p^{\mathrm{FU}} p^{\mathrm{DF}}+p^{\mathrm{HU}} p^{\mathrm{DH}}$ and $p^{\mathrm{U}}=$ $p^{\mathrm{FU}} p^{\mathrm{UF}}+p^{\mathrm{HU}} p^{\mathrm{UH}}$. Hence, for a given UE number $\tilde{k}, M^{\mathrm{FFU}}$, $M^{\mathrm{FHU}}, M^{\mathrm{D}}$ and $M^{\mathrm{U}}$ follow multinomial distributions, i.e., $M^{\mathrm{FFU}}, M^{\mathrm{FHU}}, M^{\mathrm{D}}, M^{\mathrm{U}} \sim \mathrm{M}\left(\tilde{k}, p^{\mathrm{FFU}}, p^{\mathrm{FHU}}, p^{\mathrm{D}}, p^{\mathrm{U}}\right)$. More specifically, the PMF of $M^{\mathrm{FFU}}, M^{\mathrm{FHU}}, M^{\mathrm{D}}$ and $M^{\mathrm{U}}$ can be written as

$$
\begin{aligned}
& f_{M^{\mathrm{FFU}}, M^{\mathrm{FHU}}, M^{\mathrm{D}}, M^{\mathrm{U}}}\left(m^{\mathrm{FFU}}, m^{\mathrm{FHU}}, m^{\mathrm{D}}, m^{\mathrm{U}}\right) \\
& =\frac{\tilde{k} !}{m^{\mathrm{FFU}} ! m^{\mathrm{FHU}} ! m^{\mathrm{D} !} ! m^{\mathrm{U}} !} \\
& \times\left(p^{\mathrm{FFU}}\right)^{m^{\mathrm{FE}}}\left(p^{\mathrm{FHU}}\right)^{m^{\mathrm{FHU}}}\left(p^{\mathrm{D}}\right)^{m^{\mathrm{D}}}\left(p^{\mathrm{U}}\right)^{m^{\mathrm{U}}} .
\end{aligned}
$$

\section{The Distribution of the Mode Selection in an Active BS}

After obtaining the PMF of the DL/UL data request number in an active BS, we can further derive the distribution of the mode selection in an active $\mathrm{BS}$, i.e., $q^{Q}$. In this subsection, we present our main results on $q^{Q}$ in Theorem 1 - 4 .

Theorem 1. The probability of an active BS selecting the FS mode is given by

$$
q^{\mathrm{FS}}=\sum_{\tilde{k}=1}^{+\infty}\left(1-\left(1-p^{\mathrm{FFU}}\right)^{\tilde{k}}\right) f_{\tilde{K}}(\tilde{k}),
$$

where $f_{\tilde{K}}(\tilde{k})$ is given by (4).

Proof: Conditioned on $\tilde{K}=\tilde{k}, q_{\tilde{k}}^{\mathrm{FS}}=$ $1-f_{M^{\mathrm{FFU}}, M^{\mathrm{FHU}}, M^{\mathrm{D}}, M^{\mathrm{U}}}\left(0, m^{\mathrm{FHU}}, m^{\mathrm{D}}, m^{\mathrm{U}}\right) \quad=$ $1-\left(1-p^{\mathrm{FFU}}\right)^{\tilde{k}}$. The unconditional probability is $q^{\mathrm{FS}}=\sum_{\tilde{k}=1}^{+\infty} q_{\tilde{k}}^{\mathrm{FS}} f_{\tilde{K}}(\tilde{k})$.

Theorem 2. The probability of an active BS selecting the FP mode is given by

$$
q^{\mathrm{FP}}= \begin{cases}0 & \tilde{k}=1 \\ \sum_{\tilde{k}=2}^{+\infty}\left(\left(1-p^{\mathrm{FFU}}\right)^{\tilde{k}}\right. & \tilde{k}>1 \\ \left.-\left(p^{\mathrm{D}}\right)^{\tilde{k}}-\left(p^{\mathrm{U}}\right)^{\tilde{k}}\right) f_{\tilde{K}}(\tilde{k}) & \end{cases}
$$

where $f_{\tilde{K}}(\tilde{k})$ is given by (4).

Proof: Conditioned on $\tilde{K}=\tilde{k}$, for $\tilde{k}=1, q^{\mathrm{FP}}=0$ because there is only one active UE in the cell and it is impossible for the active $\mathrm{BS}$ to find a UE pair for FD communications. For $\tilde{k}>1$, the probability of the active BS selecting FD mode is given by

$$
\begin{aligned}
& q_{\tilde{k}}^{\mathrm{FS}}+q_{\tilde{k}}^{\mathrm{FP}} \\
& =1-f_{M^{\mathrm{FFU}}, M^{\mathrm{FHU}}, M^{\mathrm{D}}, M^{\mathrm{U}}}\left(m^{\mathrm{FFU}}, m^{\mathrm{FHU}}, \tilde{k}, m^{\mathrm{U}}\right) \\
& -f_{M^{\mathrm{FFU}}, M^{\mathrm{FHU}}, M^{\mathrm{D}}, M^{\mathrm{U}}}\left(m^{\mathrm{FFU}}, m^{\mathrm{FHU}}, m^{\mathrm{D}}, \tilde{k}\right) \\
& =1-\left(p^{\mathrm{D}}\right)^{\tilde{k}}-\left(p^{\mathrm{U}}\right)^{\tilde{k}} \text {. }
\end{aligned}
$$


The unconditional probability is

$$
\begin{aligned}
& q^{\mathrm{FS}}+q^{\mathrm{FP}} \\
& =\sum_{\tilde{k}=2}^{+\infty}\left(q_{\tilde{k}}^{\mathrm{FS}}+q_{\tilde{k}}^{\mathrm{FP}}\right) f_{\tilde{K}}(\tilde{k}) \\
& =\sum_{\tilde{k}=2}^{+\infty}\left(1-\left(p^{\mathrm{D}}\right)^{\tilde{k}}-\left(p^{\mathrm{U}}\right)^{\tilde{k}}\right) f_{\tilde{K}}(\tilde{k}) .
\end{aligned}
$$

Plugging $q^{\mathrm{FS}}$ given by (7) into (10) we can derive (8).

Theorem 3. The probability of an active BS selecting the $H D$ mode is given by

$$
q^{\mathrm{HD}}=\left\{\begin{array}{ll}
p^{\mathrm{FHU}}+p^{\mathrm{D}} & \tilde{k}=1 \\
\sum_{\tilde{k}=2}^{+\infty}\left(\left(p^{\mathrm{D}}\right)^{\tilde{k}}\right) f_{\tilde{K}}(\tilde{k}) & \tilde{k}>1
\end{array},\right.
$$

where $f_{\tilde{K}}(\tilde{k})$ is given by (4).

Proof: Considering the definition of HD mode in Subsection II-B, the proof is straightforward.

Theorem 4. The probability of an active BS selecting the $H U$ mode is given by

$$
q^{\mathrm{HU}}=\sum_{\tilde{k}=1}^{+\infty}\left(\left(p^{\mathrm{U}}\right)^{\tilde{k}}\right) f_{\tilde{K}}(\tilde{k})
$$

where $f_{\tilde{K}}(\tilde{k})$ is given by (4).

Proof: Considering the definition of HU mode in Subsection II-B, the proof is straightforward.

\section{Simulation AND Discussion}

In this section, we present numerical results to validate the accuracy of our analysis and investigate the performance impact of different parameters.

\section{A. Validation of the Results on the MAC Layer Analysis}

In Fig. 2, we plot the analytical and simulation results of the full duplex BS ratio, i.e., the sum of $q^{\mathrm{FS}}$ and $q^{\mathrm{FP}}$. The UE density is set to $\rho=300 \mathrm{UEs} / \mathrm{km}^{2}$, which leads to $q=4.05$ in (2) [6]. In addition, we assume that $p^{\mathrm{FF}}=p^{\mathrm{FH}}=\frac{1}{4}, p^{\mathrm{DF}}=$ $p^{\mathrm{DH}}=\frac{1}{2}$, and $p^{\mathrm{UF}}=p^{\mathrm{UH}}=\frac{1}{4}$. Note that our analytical results in this paper can work with any value of the MAC layer parameters. From this figure, we can see that:

- The analytical results of the full duplex BS ratio match well with the simulation results.

- The FD BS ratio increases with the increase of the FD UE ratio.

- The FD BS ratio decreases with the increase of the BS density. This is because higher BS density leads to lower UE number per active BS, thus the probability of FD traffic existing decreases.

Since our analytical results match the simulation results well, we will only use analytical results of the FD BS ratio in our discussion hereafter.

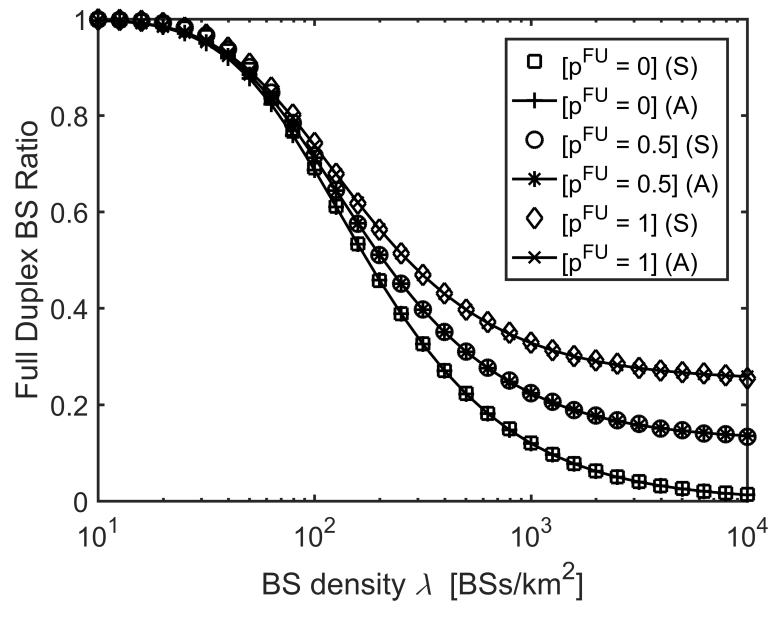

Fig. 2. The full duplex BS ratio.

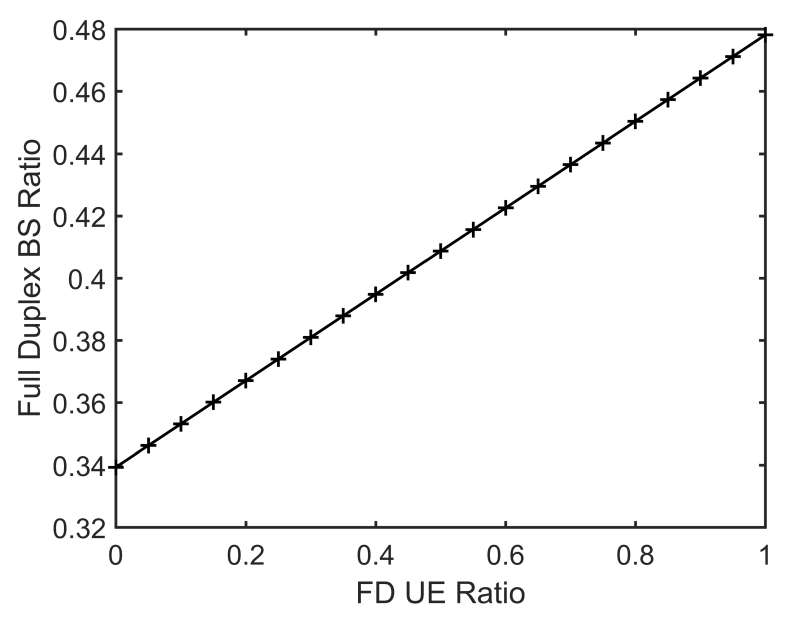

Fig. 3. The full duplex BS ratio vs. the FD UE ratio $p^{\mathrm{FU}}$.

\section{B. Discussion of the Performance Impact of FD UE Ratio $p^{\mathrm{FU}}$}

In Fig. 3, we plot the results of the FD BS ratio against the FD UE Ratio $p^{\mathrm{FU}}$. The BS density is set to $\lambda=300 \mathrm{BSs} / \mathrm{km}^{2}$ and the UE density is set to $\rho=300 \mathrm{UEs} / \mathrm{km}^{2}$. In addition, we assume that $p^{\mathrm{FF}}=p^{\mathrm{FH}}=\frac{1}{4}, p^{\mathrm{DF}}=p^{\mathrm{DH}}=\frac{1}{2}$, and $p^{\mathrm{UF}}=p^{\mathrm{UH}}=\frac{1}{4}$. As can be observed from Fig. 3, the FD BS ratio increases linearly with the increase of $p^{\mathrm{FU}}$, and thus we may expect a linear gain in the MAC layer performance with the increase of FD UE ratio.

\section{Discussion of the Performance Impact of FD Data Request Probability}

In Fig. 4, we plot the results of the FD BS ratio against the FD data request probability. The BS density is set to $\lambda=300 \mathrm{BSs} / \mathrm{km}^{2}$ and the UE density is set to $\rho=$ $300 \mathrm{UEs} / \mathrm{km}^{2}$.We assume that for FD UE and HD UE, the FD data request probability is the same, i.e., $p^{\mathrm{FF}}=p^{\mathrm{FH}}$. In addition, we assume that for each active UE, the HD-DL data request probability is twice of the HD-UL data request probability, i.e., $p^{\mathrm{DF}} / p^{\mathrm{UF}}=2 / 1$ and $p^{\mathrm{DH}} / p^{\mathrm{UH}}=2 / 1$. From 


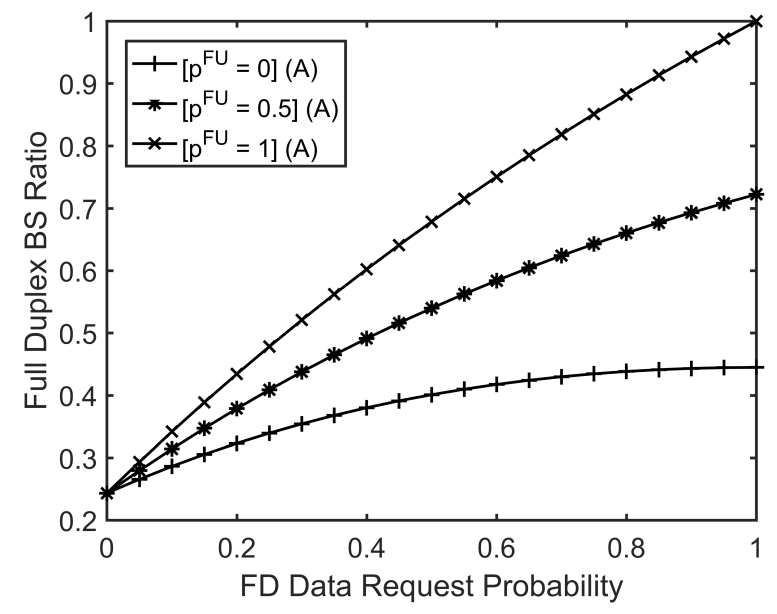

Fig. 4. The full duplex BS ratio vs. the FD data request probability.

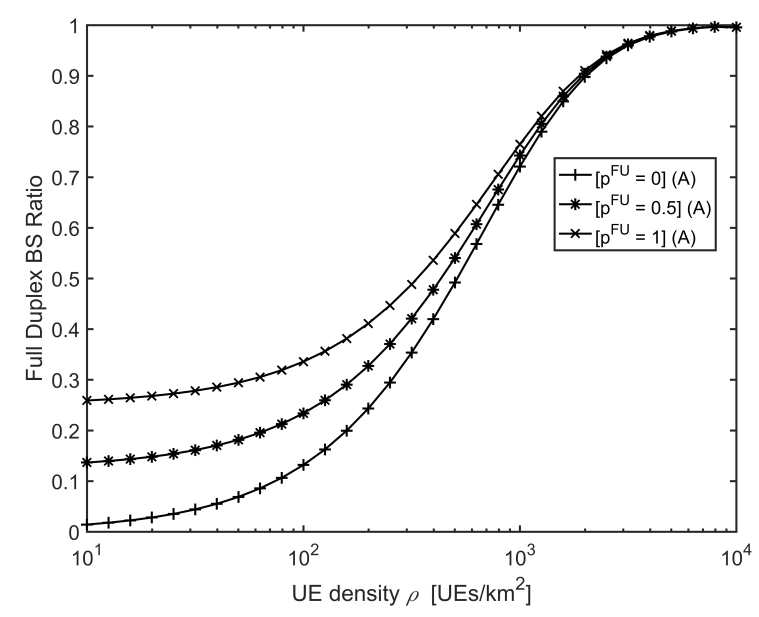

Fig. 5. The full duplex BS ratio vs. the UE density $\rho$.

Fig. 4, we can see that:

- The FD BS ratio increases with the increase of FD data request probability.

- The increasing speed of the FD BS ratio gradually slows down with the increase of FD data request probability. Moreover, the increasing speed of the FD BS ratio with a larger FD UE Ratio $p^{\mathrm{FU}}$ lessens slower with the increase of FD data request probability, thanks to the contribution of FS mode with FD UE.

\section{Discussion of the Performance Impact of UE Density $\rho$}

In Fig. 5, we plot the results of the FD BS ratio against the UE density $\rho$. The BS density is set to $\lambda=300 \mathrm{BSs} / \mathrm{km}^{2}$, besides, we assume that $p^{\mathrm{FF}}=p^{\mathrm{FH}}=\frac{1}{4}, p^{\mathrm{DF}}=p^{\mathrm{DH}}=\frac{1}{2}$, and $p^{\mathrm{UF}}=p^{\mathrm{UH}}=\frac{1}{4}$. From Fig. 5, we can see that:

- For different FD UE ratio $p^{\mathrm{FU}}$, the FD BS ratio first increases gradually with the increase of UE density $\rho$, then picks up the increasing speed, and finally converges to 1 when $\rho$ becomes large enough.
- For different FD UE ratio $p^{\mathrm{FU}}$, the gap of the FD BS ratio decreases with the increase of UE density $\rho$.

\section{CONCLUSion}

For the first time, we analytically study the MAC layer performance of FD in a realistic SCN scenario, where BSs can select FD/HD mode according to the real-time DL/UL traffic. The analytical results are shown to match the simulation results well. As our future work, we will combine our results on the MAC layer BS mode selection with the PHY layer SINR results to derive the total area spectral efficiency for FD.

\section{REFERENCES}

[1] D. López-Pérez, M. Ding, H. Claussen, and A. Jafari, "Towards 1 Gbps/UE in cellular systems: Understanding ultra-dense small cell deployments," IEEE Communications Surveys Tutorials, vol. 17, no. 4, pp. 2078-2101, Jun. 2015.

[2] G. Yu, Z. Zhang, F. Qu, and G. Y. Li, "Ultra-dense heterogeneous networks with full-duplex small cell base stations," IEEE Network, vol. 31, no. 6, pp. 108-114, November 2017.

[3] J. Liu, S. Han, W. Liu, and C. Yang, "The value of full-duplex for cellular networks: A hybrid duplex-based study," IEEE Transactions on Communications, vol. 65, no. 12, pp. 5559-5573, Dec 2017.

[4] I. Atzeni and M. Kountouris, "Full-duplex mimo small-cell networks with interference cancellation," IEEE Transactions on Wireless Communications, vol. 16, no. 12, pp. 8362-8376, Dec 2017.

[5] D. Korpi, J. Tamminen, M. Turunen, T. Huusari, Y. S. Choi, L. Anttila, S. Talwar, and M. Valkama, "Full-duplex mobile device: pushing the limits," IEEE Communications Magazine, vol. 54, no. 9, pp. 80-87, September 2016.

[6] M. Ding, D. López-Pérez, G. Mao, and Z. Lin, "Performance impact of idle mode capability on dense small cell networks," IEEE Transactions on Vehicular Technology, vol. 66, no. 11, pp. 10446-10460, Nov 2017.

[7] M. Ding, D. López-Pérez, and G. Mao, "A new capacity scaling law in ultra-dense networks," arXiv:1704.00399 [cs.NI], Apr. 2017. [Online]. Available: https://arxiv.org/abs/1704.00399

[8] S. Lee and K. Huang, "Coverage and economy of cellular networks with many base stations," IEEE Communications Letters, vol. 16, no. 7, pp. 1038-1040, Jul. 2012.

[9] M. Ding, D. Lopez Perez, G. Mao, and Z. Lin, "Study on the idle mode capability with LoS and NLoS transmissions," arXiv:1608.06694 [cs.NI], to appear in IEEE Globecom 2016, Dec. 2016.

[10] I. Gradshteyn and I. Ryzhik, Table of Integrals, Series, and Products (7th Ed.). Academic Press, 2007. 